\title{
Adiabatic Reverse Combustion in a Packed Bed
}

\author{
M. FATEHI and M. KAVIANY \\ Department of Mechanical Engineering and Applied Mechanics, University of Michigan, Ann Arbor, MI 48109
}

\begin{abstract}
Downward propagation of a combustion front in a packed bed of wood particles, with air supply from below, is examined theoretically and experimentally. Using a single-step reaction, with a kinetic model of char oxidation as the dominant mechanism, and assuming local thermal equilibrium, but allowing a local chemical nonequilibrium between the solid and gas phases, the front speed $u_{F}$, the adiabatic temperature $T_{r}$, and the extent of solid consumption $\langle\rho\rangle_{r}^{s} /\langle\rho\rangle_{n}{ }^{s}$ are determined as functions of the entering air pole-velocity $\langle u\rangle_{n}^{g}$. Both oxygen-limited and fuel-limited regimes, with the boundary marked by the stoichiometric burning, are examined. In the oxygen-limited regime, $T_{r}, u_{F},\langle\rho\rangle_{r}^{s}\left\langle\langle\rho\rangle_{n}^{s}\right.$, and the thickness of the front $\delta_{F}$ all increase with $\langle u\rangle_{n}{ }^{g}$. In the fuel-limited regime, the reverse occurs and the extinction at high $\langle u\rangle_{n}{ }^{g}$ is predicted. In the oxygen-limited regime, where the bed is not yet fluidized, the experimental results are in good agreement with the predictions.
\end{abstract}

\section{NOMENCLATURE}

$a_{1} \quad$ pre-exponential factor $\left(\mathrm{s}^{-1}\right)$

Asg solid-gas surface area $\left(\mathrm{m}^{-1}\right)$

$c_{p} \quad$ specific heat capacity $(\mathrm{J} / \mathrm{kg}-\mathrm{K})$

$d \quad$ diameter $(\mathrm{m})$

$D \quad$ total thermal diffusivity $\left(\mathrm{m}^{2} / \mathrm{s}\right)$

$D_{m} \quad$ mass diffusivity $\left(\mathrm{m}^{2} / \mathrm{s}\right)$

$D^{d} \quad$ axial thermal dispersion coefficient $\left(\mathrm{m}^{2} / \mathrm{s}\right)$

$\Delta E_{a} \quad$ activation energy for reaction $(\mathrm{J} / \mathrm{mole})$

$F \quad$ radiation exchange factor

$\Delta i_{c} \quad$ heat of combustion $(\mathrm{J} / \mathrm{kg})$

$k$ thermal conductivity $(\mathrm{W} / \mathrm{m}-\mathrm{K})$

$K \quad$ kinetic rate $\left(\mathrm{s}^{-1}\right)$

$\dot{n}_{d} \quad$ diffusion-controlled reaction rate $\left(\mathrm{kg} / \mathrm{m}^{3} \mathrm{~s}\right)$

$\dot{n}_{\Delta E} \quad$ kinetically controlled reaction rate $\left(\mathrm{kg} / \mathrm{m}^{3}-\mathrm{s}\right)$

$\dot{n} \quad$ reaction rate $\left(\mathrm{kg} / \mathrm{m}^{3}-\mathrm{s}\right)$

$\mathrm{Le}_{g} \quad$ gas Lewis number

$\mathrm{Pe}_{d} \quad$ Péclet number

$R_{g} \quad$ gas constant $(\mathrm{J} /$ mole- $\mathrm{K})$

Sc Schmidt number

$\left\langle\mathrm{Sh}_{d}\right\rangle \mathrm{sg}$ Sherwood number $t$

$T$

$u$

V

time (s)

temperature $(K)$

velocity $(\mathrm{m} / \mathrm{s})$

representative elementary volume $\left(\mathrm{m}^{3}\right)$

$x \quad$ spatial coordinate (m)

$x_{1} \quad$ moving spatial coordinates $(\mathrm{m})$

\section{Greek Symbols}

$\alpha \quad$ thermal diffusivity $\left(\mathrm{m}^{2} / \mathrm{s}\right)$

$\epsilon$ porosity

$\epsilon_{r} \quad$ surface emissivity

$\delta_{F} \quad$ combustion-front thickness (m)

$\rho \quad$ density $\left(\mathrm{kg} / \mathrm{m}^{3}\right)$

$\sigma \quad$ Stefan-Boltzmann constant $\left(\mathrm{W} / \mathrm{m}^{2}\right.$ $\mathrm{K}^{4}$ )

$\nu \quad$ kinematic viscosity $\left(\mathrm{m}^{2} / \mathrm{s}\right)$

\section{Superscripts}

$\begin{array}{ll}d & \text { dispersion } \\ g & \text { gas } \\ s & \text { solid }\end{array}$

\section{Subscripts}

$a$

$c$

$d$

ash or activation

char

diffusion

$D$ Darcean

$e \quad$ effective

$F$ front

$g \quad$ gas

$g p \quad$ gaseous products

$i$ initial

$n$ nonreacted

$o$ oxygen

$r \quad$ reacted

Copyright (C) 1994 by The Combustion Institute

Published by Elsevier Science Inc.

$0010-2180 / 94 / \$ 7.00$ 
$s$

st

$t$

$v$

$x$

\section{Others}

$\begin{array}{ll}\langle\rangle & \text { volume-averaged, } V^{-1} \int_{V} \phi d V, V= \\ \langle\rangle^{g} & V_{g}+V_{s} \\ & \text { gas-phase volume-averaged, } V_{g}^{-1} \int_{V_{g}} \\ & \phi d V \\ \langle\rangle^{s} & \text { solid-phase volume-averaged, } V_{s}^{-1} \\ & \int_{V_{s}} \phi d V\end{array}$

\section{INTRODUCTION}

Combustion in noninert porous media has been the subject of numerous experimental and theoretical investigations over the last decade. This phenomenon has a wide range of technological relevance, as in the control of fire spread in permeable solids, high-temperature synthesis of materials, in situ coal gasification, and design of fixed-bed catalytic reactors (surfacereaction-dominated processes). Other areas include the packed-bed incineration of municipal solid waste, the incineration of hazardous solid waste, and biomass combustion. Chemical reactions in a porous medium are characterized by large gradients in the temperature and the species-concentrations, resulting in self-sustained propagation of the thermal wave. This phenomenon is observed when the heat released in the gas phase and/or at the gas-solid interface is transferred to the unreacted gas and solid. The speed at which the burning front propagates is primarily controlled by the gas flow rate and direction and the initial concentration of the oxidant. The air flow opposite to front propagation is referred to as the reverse combustion. At low gas flow rates, the process is limited to the surface reactions (i.e., smoldering combustion); at high gas flow rates, both homogeneous and heterogeneous reactions can occur.

Smoldering combustion of porous materials has been previously studied both experimentally and theoretically, usually in the context of fire safety. An earlier review is given by Palmer [1]. The burning of cellulosic materials with the thermobuoyant flow of oxygen has been investigated by Ohlemiller [2, 3]. The transition to flaming through the forced flow of oxygen has been investigated experimentally by Ohlemiller $[4,5]$, Rogers and Ohlemiller [6], and Ohlemiller and Rogers [7]. The reverse smoldering of flexible polyurethane foams with forced flow has been examined experimentally by Rogers and Ohlemiller [8]. By applying a forced flow, comparable in magnitude to the thermobuoyant flow $\left(\epsilon\langle u\rangle_{n}{ }^{g}=0.04\right.$ and $\epsilon\langle u\rangle_{n}{ }^{g}=0.15$ $\mathrm{cm} / \mathrm{s}$, where $\epsilon$ is the porosity and $\langle u\rangle_{n}{ }^{g}$ is the nonreacted, inlet gas pore-velocity) they conclude that increased smolder velocities $u_{F}$ are accompanied with higher final temperatures $T_{r}$, thinner smolder waves, and increased solid conversion (to the gaseous products and ash) $\langle\rho\rangle_{r}^{s} /\langle\rho\rangle_{n}{ }^{s}$. Robinovich and Gurevich [9] have modeled the forced-flow burning of combustible porous media in which the products were in a condensed phase. No melting of the solid phase (reactants or products) was incorporated, but the effect of a lateral heat loss on $u_{F}$ and the extent of the conversion of the original reactants to the final solid products was addressed. Dosanjh et al. [10] and Torero et al. [11], using reverse and forward combustion, respectively, have investigated a one-step reaction model for the oxidation and pyrolysis of the solid. Ohlemiller and Lucca [12] have studied both forward and reverse smoldering in cellulosic loose fill insulations, and a particulate polymer material (polyisocyanurate). They have observed marked qualitative differences in the two smolder propagation modes. Reverse smolder quickly reaches a steady propagation rate determined largely by the axial heat transfer rate, whereas forward smolder is unsteady and moves at a higher rate, which appears to be limited by the stoichiometry of the char oxidation. Both modes of propagation were ultimately limited by the rate of oxygen supply. Moussa et al. [13] have experimentally and theoretically investigated the mechanism of smoldering in cellulosic materials. In steady smoldering, the measured spreading speed and the maximum temperature of the smoldering zone were found to depend on the ambient oxygen mole fraction and partial pressure. However, independent of these parameters, the speed was determined to be uniquely related 
to the maximum temperature. Their analysis treated the cellulose pyrolysis and char oxidation by a two-zone reaction model separated by an interface, with the appropriate matching requirements.

Most studies on the propagation of the burning front in combustible porous media have considered low oxidant flow rates (i.e., smoldering combustion, which is in the oxygenlimited regime). At high oxidant flow rates, both the heterogeneous surface reactions and the homogeneous gas-phase reactions are observable. These reactions compete in the consumption of the available oxidant according to their respective kinetics. However, separate description of these kinetics has proved difficult. As the oxidant flow rate is further increased, a fuel-limited regime is encountered. In this study, we consider arbitrary oxidant flow rates. Assumed is local thermal equilibrium, but allowed is local chemical nonequilibrium between the solid and gas phases. Despite the use of simplified reaction kinetics (assumed dominated by surface oxidation), the overall features of the combustion process observed in the experiment, i.e., $T_{r}, u_{F}$, and $\langle\rho\rangle_{r}^{s} /\langle\rho\rangle_{n}^{s}$ have been accurately predicted.

\section{ANALYSIS}

\section{Problem Definition and Formulation}

Figure 1 schematically shows the structure of the adiabatic reverse-combustion process modeled. The incoming oxidizer, air $\left(\left\langle\rho_{0}\right\rangle_{n}^{g} /\langle\rho\rangle_{n}{ }^{g}\right.$ $=0.23$ ), flows in a direction opposite to that of the reaction front. Exothermic reactions occurring in the combustion zone supply the thermal energy required to sustain the burning front. This energy is dominantly transported upstream via radiation and conduction through the solid (from the combustion zone to the nonreacted gas and solid). Solid endothermic reactions result in the pyrolytic formation of char, and endothermic gasification releases volatiles into the gas phase. Therefore, in addition to the direct attack of the oxygen on the surface, depending on the temperature, both gas-phase oxidation and char oxidation occur, and should be addressed. A three-step reaction model for surface heterogeneous reactions, which will allow for char formation and oxidation, is given by Rogers and Ohlemiller $[6,8]$, as follows:

\section{Endothermic Pyrolysis}

1 g(Fuel)

$$
\begin{aligned}
& \rightarrow \nu_{c 1}(\text { Char }) \\
& \quad+\left(1-\nu_{c 1}\right)(\text { Gaseous Products } 1)
\end{aligned}
$$

\section{Exothermic Thermal Oxidation}

$$
\begin{aligned}
1 \mathrm{~g}(\text { Fuel }) & +\nu_{o 2} \mathrm{O}_{2} \\
\rightarrow & \nu_{c 2}(\text { Char }) \\
& +\left(1+\nu_{o 2}-\nu_{c 2}\right)(\text { Gaseous Products } 2)
\end{aligned}
$$

\section{Exothermic Char Oxidation}

$$
\begin{aligned}
1 \mathrm{~g}(\mathrm{Char}) & +\nu_{o 3} \mathrm{O}_{2} \\
\rightarrow & \nu_{a 3}(\text { Ash }) \\
& +\left(1+\nu_{o 3}-\nu_{a 3}\right) \text { (Gaseous Products 3). }
\end{aligned}
$$

Reaction given by Eq. 2 is expected to be less significant for large wood particles, where the oxygen diffusion through the particle is the limiting mechanism. However, it can become significant (and even dominant) for small particles.

A simplified kinetic model that assumes a one-step global reaction has been used by Dosanjh et al. [10], as follows:

$$
\begin{aligned}
& 1 \mathrm{~g} \text { (Unburned Solid) }+\nu_{o} \mathrm{O}_{2} \\
& \rightarrow \nu_{a} \text { (Ash) } \\
& +\nu_{g p} \text { (Gaseous Products) }+\frac{\Delta i_{c}}{\nu_{o}} \dot{n}_{o},
\end{aligned}
$$

Assumed in Eq. 4 is that pyrolysis kinetics is much faster than char oxidation, and that the heat released from char oxidation is much higher than the endothermicity of the pyrolytic reactions. Following Dosanjh et al. [10], these assumptions allow the three-step reaction mechanism for pyrolysis, oxidative thermal 


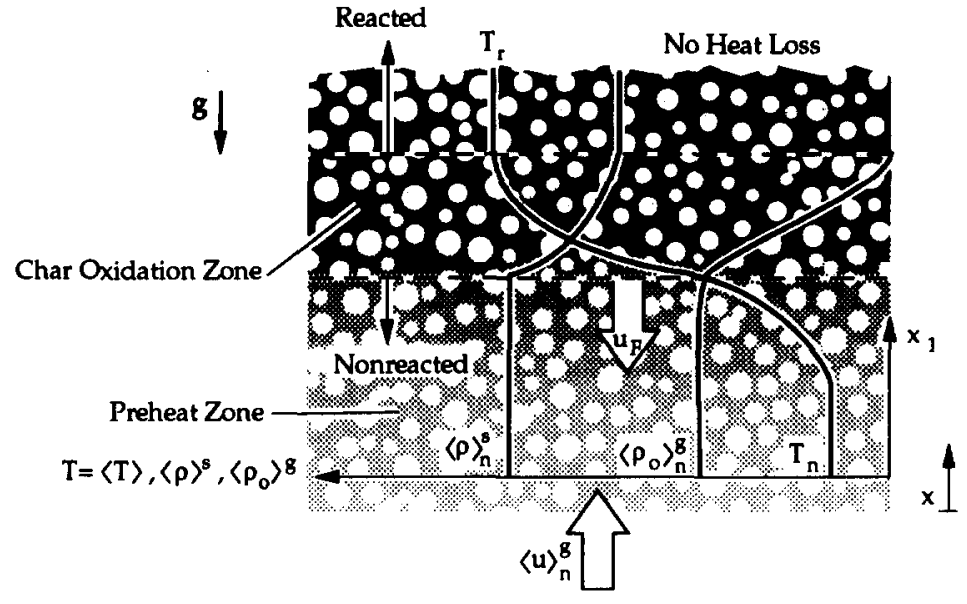

Fig. 1. A schematic of the structure of the adiabatic, planar combustion front traveling through a packed bed. The fixed and moving coordinates are shown. degradation, and char oxidation to be combined into the one-step reaction model.

In modeling the quasi-steady, one-dimensional adiabatic burning front propagating through the medium, it was assumed that the gas and solid were in a local thermal equilibrium. Then using local volume averaging principles, governing equations were written assuming that the front thickness $\delta_{F}$ was much larger than the particle size, $\delta_{F} / d \gg 1$. The local volume-averaged energy equation becomes

$$
\begin{gathered}
{\left[\epsilon\left\langle\rho c_{p}\right\rangle^{g}+(1-\epsilon)\left\langle\rho c_{p}\right\rangle^{s}\right] \frac{\partial T}{\partial t}} \\
+\epsilon\left\langle\rho c_{p}\right\rangle^{g}\langle u\rangle^{g} \frac{\partial T}{\partial x} \\
=\frac{\partial}{\partial x}\left[\left(\langle k\rangle+\left\langle k_{r}\right\rangle\right) \frac{\partial T}{\partial x}\right] \\
+\left(\frac{\Delta i_{c}}{\nu_{o}}\right)\left\langle\dot{n}_{o}\right\rangle,
\end{gathered}
$$

where it has been assumed that

$$
\langle T\rangle^{s}(x, t)=\langle T\rangle^{g}(x, t)=T(x, t) .
$$

In Eq. 5, the total thermal conductivity $\langle k\rangle$, which is the sum of the molecular and axial hydrodynamic dispersion components, is expressed as

$\langle k\rangle=k_{e}+\epsilon\left(\rho c_{p}\right)_{g} D^{d}$.
Since the solid is stationary $\left(\langle u\rangle^{s}=0\right)$, a coordinate axis $x_{1}$, fixed at the combustion front and a constant front speed $u_{F}$ are assumed, i.e., the front travels at a steady speed $u_{F}=$ $(1-\epsilon)\langle u\rangle_{F}^{s}$, and

$x_{1}=x+u_{F} t$.

Then Eq. 5 is transformed to

$$
\begin{aligned}
\frac{d}{d x_{1}}\left[(1-\epsilon)\left\langle\rho c_{p}\right\rangle^{s} u_{F} T+\left\langle\rho c_{p}\right\rangle^{g} u_{D}\right] T \\
=\frac{d}{d x_{1}}\left[\left(\langle k\rangle+\left\langle k_{r}\right\rangle\right) \frac{d T}{d x_{1}}\right] \\
+\left(\frac{\Delta i_{c}}{\nu_{o}}\right)\left\langle\dot{n}_{o}\right\rangle
\end{aligned}
$$

where the relative, gas-phase Darcean velocity is defined as

$$
u_{D}=\epsilon\left(\langle u\rangle^{g}+u_{F}\right)
$$

The pressure drop across the burning front was assumed negligible (i.e., a high permeability was assumed). The momentum equation, i.e., the Darcy law, then becomes trivial. In this coordinate system, the local volume-averaged conservation equations for the overall gasphase mass, for the $\mathrm{O}_{2}$ in the gas, and for the 
unburned fuel in the solid, are

$$
\begin{aligned}
& \frac{d}{d x_{1}}\left(\langle\rho\rangle^{g} u_{D}\right)=\left\langle\dot{n}_{o}\right\rangle+\left\langle\dot{n}_{g p}\right\rangle \\
& \frac{d}{d x_{1}}\left(u_{D}\left\langle\rho_{o}\right\rangle^{g}\right) \\
& \quad=\frac{d}{d x_{1}}\left(\epsilon\langle\rho\rangle^{g}\left\langle D_{m}\right\rangle \frac{d}{d x_{1}} \frac{\left\langle\rho_{o}\right\rangle^{g}}{\langle\rho\rangle^{g}}\right)+\left\langle\dot{n}_{o}\right\rangle \\
& \frac{d}{d x_{1}}\left[(1-\epsilon) u_{F}\langle\rho\rangle^{s}\right]=\left\langle\dot{n}_{s}\right\rangle .
\end{aligned}
$$

Note that the ash products are not included in $\langle\rho\rangle^{s}$. The reaction rate for the consumption of oxygen species at the solid surface (heterogeneous reaction) is modeled as a one-step, first-order Arrhenius kinetics:

$$
\left.\dot{n}_{\Delta E}=-a_{1} \nu_{o} \rho_{o, s}\langle\rho\rangle\right\rangle^{s-\Delta E_{a} / R_{g} T} \text { on } A_{s g} .
$$

By considering local chemical nonequilibrium between the gas and solid phases (i.e., the concentration of $\mathrm{O}_{2}$ in the gas phase differs from that at the solid surface), the local volume-averaged reaction rate is, in part, controlled by the rate at which the oxygen can diffuse to the surface of the solid particles. This is modeled using a solid-gas mass transfer coefficient (i.e., Sherwood number $\left\langle\mathrm{Sh}_{d}\right\rangle_{A s g}$ ). The rate of reaction is then modeled by Eq. 14, a surface kinetic-controlled reaction, which is in series with a mass transfer control reaction given by:

$$
\begin{aligned}
\dot{n}_{d}= & -\frac{\left\langle\mathrm{Sh}_{d}\right\rangle_{A_{s g}} D_{m, g}}{\epsilon d} \frac{A_{s g}}{V} \\
& \times\left(\rho_{o}-\rho_{o, s}\right) \text { on } A_{s g},
\end{aligned}
$$

$\rho_{o} \gg \rho_{o, s}$, fast kinetics.

The local volume-averaged reaction rate is therefore modified as [14]

$$
\left\langle\dot{n}_{o}\right\rangle=\frac{1}{\frac{1}{\dot{n}_{d}}+\frac{1}{\dot{n}_{\Delta E}}} .
$$

Based on the one-step global reaction of Eq. 4 , the remaining reaction rates can be written as

$$
\left\langle\dot{n}_{g p}\right\rangle=-v_{g p}\left\langle\dot{n}_{o}\right\rangle,\left\langle\dot{n}_{s}\right\rangle=\frac{1}{\nu_{o}}\left\langle\dot{n}_{o}\right\rangle .
$$

In comparison to $\dot{n}_{d}$, the kinetically controlled reaction rate, expressed by Eq. 14, becomes significant when the temperature is high or the particle diameter is small $\left(\rho_{o} \simeq \rho_{o, s}\right)$. Thus, the following two distinct regimes of reaction can be identified:

$$
\dot{n}_{\Delta E} \gg \dot{n}_{d} \Rightarrow\left\langle\dot{n}_{o}\right\rangle=\dot{n}_{d} \quad \text { diffusion controlled, }
$$

$$
\begin{aligned}
\dot{n}_{\Delta E} \ll \dot{n}_{d} \Rightarrow & \left\langle\dot{n}_{o}\right\rangle \\
& =\dot{n}_{\Delta E} \quad \text { kinetically controlled. }
\end{aligned}
$$

In Eq. 15, the specific surface area for a packed bed composed of spherical particles $A_{s g} / V$ is equal to $6(1-\epsilon) / d$. The interstitial mass transfer Sherwood number is taken as [15]:

$\left\langle\mathrm{Sh}_{d}\right\rangle_{A_{s} g}=2+1.1 \operatorname{Re}_{d}{ }^{0.6} \mathrm{Sc}^{0.33}$.

The Reynolds and Schmidt numbers are defined as $\operatorname{Re}_{d}=u_{D} d / \nu_{g}$ and $\mathrm{Sc}=\nu_{g} / D_{m, g}$, respectively; $D_{m, g}$ is the molecular mass diffusivity of the gas phase.

The total thermal diffusivity of the medium is defined as [16]

$D=\frac{k_{e}}{\left(\rho c_{p}\right)_{g}}+\epsilon D^{d}$,

where

$\frac{k_{e}}{k_{g}}=\left(\frac{k_{s}}{k_{g}}\right)^{0.280-0.757 \log \epsilon-0.057 \log \left(k_{s} / k_{g}\right)}$

The axial thermal dispersion coefficient $D^{d}$, which is a function of the Péclet number $\mathrm{Pe}_{t}$ based on the particle radius, is given by

$$
\begin{aligned}
D^{d} & =\alpha_{g}\left[\frac{3}{4} \mathrm{Pe}_{t}+\frac{1}{6} \pi^{2}(1-\epsilon) \mathrm{Pe}_{t} \ln \mathrm{Pe}_{t}\right], \\
\mathrm{Pe}_{t} & =\frac{u_{D} d}{2 \alpha_{g}} .
\end{aligned}
$$


The radiative exchange was assumed to occur among the opaque surface of the particles and the gas was treated as nonparticipating, i.e., radiative energy was emitted by the particle surface and was absorbed and scattered by the particles. By treating the packed bed as a continuum at the optically thick limit, radiative conductivity $\left\langle k_{r}\right\rangle$ models the radiative heat flux through the medium. The radiant conductivity is expressed as

$$
\left\langle k_{r}\right\rangle=4 F d \sigma T^{3} \text {. }
$$

For opaque spherical particles, the radiant exchange factor $F$ depends on $k_{s}, \epsilon$, surface radiation properties (such as emissivity $\epsilon_{r}$ ) and the extent of specular reflection. The radiation exchange factor $F$ has been determined by Singh and Kaviany [17] and is given by the following correlation:

$$
\begin{aligned}
F & =k_{r}^{*}=\frac{k_{r}}{4 d \sigma T^{3}} \\
& =a_{F 1} \epsilon_{r} \tan ^{-1}\left(a_{F_{2}} \frac{k_{s}^{* a_{F_{3}}}}{\epsilon_{r}}\right)+a_{F_{4}}, \\
k_{s}^{*} & =\frac{k_{s}}{4 d \sigma T^{3}}
\end{aligned}
$$

For diffuse surfaces, values for $a_{F_{i}}$ are 0.5756 , $1.535,0.8011$, and 0.1843 .

The total mass diffusivity in the porous medium is similarly defined as [16]

$$
\left\langle D_{m}\right\rangle=D_{m e}+D_{m}^{d},
$$

where

$D_{m e}=\frac{2 \epsilon}{3-\epsilon} D_{m, g}$

The axial mass dispersion coefficient $D_{m}{ }^{d}$ is defined similar to $D^{d}$ as

$$
\begin{aligned}
D_{m}{ }^{d}= & D_{m, g} \\
& \times\left[\frac{3}{4} \mathrm{Pe}_{m}+\frac{1}{6} \pi^{2}(1-\epsilon) \mathrm{Pe}_{m} \ln \mathrm{Pe}_{m}\right],
\end{aligned}
$$

where the mass Péclet number is defined as

$$
\mathrm{Pe}_{m}=\frac{u_{D} d}{2 D_{m, g}}
$$

\section{Asymptotic Solution}

The method of large activation energy asymptotics, which was originally applied by Dosanjh et al. [10] to this problem, was used here to obtain the adiabatic temperature $T_{r}$ and the front speed $u_{F}$. It is assumed that $\epsilon_{o}=$ $\Delta E_{a} / R_{g} T_{r} \gg 1$, and that $\langle\rho\rangle^{g},\langle\rho k\rangle^{g}$, $\left\langle k_{r}\right\rangle\left(T_{r}\right)=4 F d \sigma T_{r}^{3}$, and $\left\langle c_{p}\right\rangle^{g}=\left\langle c_{p}\right\rangle^{s}=c_{p}$ were constant and $u_{D} \gg u_{F}$. The single-energy and species equations for the gas and solid phases, in dimensionless form, are as follows:

$$
\begin{aligned}
\frac{d T^{*}}{d x^{*}}=[ & \left.1+R_{c}\left(1+\phi T^{*}\right)^{3}\right] \frac{d^{2} T^{*}}{d x^{* 2}} \\
& +3 R_{c}\langle k\rangle \phi\left(1+\phi T^{*}\right)^{2}\left(\frac{d T^{*}}{d x^{*}}\right)^{2} \\
& -\Gamma^{\prime}\left(1-\rho_{o}^{*}\right)\left(1-\rho_{s}^{*}\right) e^{-\Delta E_{a} / R_{g} T},
\end{aligned}
$$

$$
\begin{aligned}
\frac{d \rho_{o}^{*}}{d x_{1}^{*}}= & \frac{1}{\operatorname{Le}_{g}} \frac{d^{2} \rho_{o^{*}}}{d x_{1}^{* 2}} \\
& +\Gamma^{\prime \prime}\left(1-\rho_{o}^{*}\right)\left(1-\rho_{s}^{*}\right) e^{-\Delta E_{a} / R_{g} T},
\end{aligned}
$$

$$
\frac{d \rho_{s}^{*}}{d x_{1}^{*}}=\Gamma^{\prime \prime \prime}\left(1-\rho_{o}^{*}\right)\left(1-\rho_{s}^{*}\right) e^{-\Delta E_{a} / R_{g} T}
$$

The boundary conditions are

$$
\begin{aligned}
& T^{*}=\frac{d T^{*}}{d x^{*}}=0, \quad x^{*} \rightarrow-\infty, \\
& T^{*}=1, \quad \frac{d T^{*}}{d x^{*}}=0, \quad x^{*} \rightarrow 0, \\
& \rho_{o}^{*}=0, \quad \frac{d \rho_{o}^{*}}{d x_{1}^{*}}=\rho_{s}^{*}=\frac{d \rho_{s}^{*}}{d x_{1}^{*}}=0 \quad x_{1}^{*} \rightarrow-\infty
\end{aligned}
$$

$$
\rho_{o}^{*}=1, \quad \frac{d \rho_{o}^{*}}{d x_{1}^{*}}=\frac{d \rho_{s}^{*}}{d x_{1}^{*}}=0, \quad x_{1}^{*} \rightarrow 0
$$


where

$$
\begin{aligned}
& x^{*}=\int \frac{\langle\rho\rangle\langle u\rangle c_{p}}{\langle k\rangle} d x_{1}, \\
& x_{1}^{*}=\int \frac{\epsilon\left\langle\rho_{g} c_{p}\right\rangle^{g}\langle u\rangle}{\langle k\rangle^{g}} d x_{1}, \\
& T^{*}=\frac{T-T_{n}}{T_{r}-T_{n}}, \quad \rho_{o}^{*}=1-\frac{\left\langle\rho_{o}\right\rangle^{g}}{\left\langle\rho_{o}\right\rangle_{n}^{g}}, \\
& \rho_{s}^{*}=1-\frac{\langle\rho\rangle^{s}}{\langle\rho\rangle_{n}^{s}}, \\
& \phi=\frac{T_{r}-T_{n}}{T_{n}}, \quad R_{c}=\frac{4 F d \sigma T_{n}^{3}}{\langle k\rangle} \\
& \mathrm{Le}_{g}=\frac{\langle k\rangle^{g}}{\epsilon\left\langle\rho c_{p}\right\rangle^{g}\left\langle D_{m}\right\rangle}, \\
& \Gamma^{\prime}=\frac{a_{1}\langle k\rangle\langle\rho\rangle_{n}^{s} \nu_{o}}{\epsilon\langle u\rangle_{n}{ }^{g}\langle\rho\rangle\langle u\rangle_{p}}, \\
& \Gamma^{\prime \prime}=\frac{a_{1} \nu_{o}\langle k\rangle^{g}\langle\rho\rangle_{n}{ }^{s}}{c_{p}\left(\epsilon\langle u\rangle_{n}^{g}\right)^{2}\left\langle\rho_{o}\right\rangle_{n}^{g}} \\
& \Gamma^{\prime \prime \prime}=\frac{a_{1}\langle k\rangle^{g}\left\langle\rho_{o}\right\rangle_{n}{ }^{g}}{\epsilon\langle u\rangle_{n}{ }^{g} c_{p} u_{F}(1-\epsilon)} .
\end{aligned}
$$

In Eq. 37, the phase mass-averaged velocity is

$$
\begin{aligned}
\langle\rho\rangle\langle u\rangle & =(1-\epsilon)\langle\rho\rangle^{s} u_{F}+\epsilon\langle\rho\rangle^{g}\langle u\rangle^{g} \\
& =\text { constant. }
\end{aligned}
$$

To employ the method of large activation asymptotics, the diffusion controlled-reaction (Eq. 15) can also be modified for first-order Arrhenius-type kinetics. $\Delta E_{d}$, the activation energy for the mass diffusion, being substituted for the chemical activation energy $\Delta E_{a}$. The ratio $\Delta E_{d} / \Delta E_{a}$ is determined empirically and can vary from 0.5 to 1 depending on the extent of diffusional control [18].

The reaction zone was defined as the region where the reaction and diffusion were dominant over the convective terms, under the condition that $\delta^{*}=1 / \epsilon_{o}=R_{g} T_{r} / \Delta E_{a} \ll 1$. A new spatial coordinate $\eta$, was then introduced which is scaled by $\delta^{*}$ :

$$
\eta=\frac{x_{1}^{*}}{\delta^{*}} .
$$

Since in the inner zone the temperature can only suffer $O(\delta)$ reduction from the leading order, the combined thermal conductivity $k_{c}$ is taken to be:

$$
k_{c}=\left\langle k_{r}\right\rangle\left(T_{r}\right)+\langle k\rangle,\left\langle k_{r}\right\rangle\left(T_{r}\right)=4 F d \sigma T_{r}^{3} .
$$

This simplified the energy equation (Eq. 30) in the inner zone by setting $R_{c}=0$. The inner solutions in terms of $\delta^{*}$ up to the first leading order were then expanded:

$$
\begin{aligned}
& \rho_{o}^{*+}=1-\delta^{*} \gamma(\eta)-O\left(\delta^{* 2}\right), \\
& T^{*+}=1-\delta^{*} \theta(\eta)-O\left(\delta^{* 2}\right), \\
& \rho_{s}^{*+}=\delta^{*} \Lambda(\eta)+O\left(\delta^{* 2}\right) .
\end{aligned}
$$

It can be shown that

$$
\gamma(\eta)=\frac{\operatorname{Le}_{g} \Gamma^{\prime \prime}}{C_{2}^{2} \Gamma^{\prime}} \theta(\eta)
$$

Similarly:

$$
\frac{d \theta(\eta)}{d \eta}=\frac{C_{2} \Gamma^{\prime} \delta^{*}}{\Gamma^{\prime \prime \prime}}\left(\Lambda-\Lambda_{1}\right),
$$

In Eq. 48, $\Lambda(\eta=0)=\Lambda_{1}$ and $C_{2}=$ $C_{1}\langle k\rangle /\langle k\rangle_{t}$. The following relations must be defined:

$$
\begin{aligned}
& \begin{aligned}
\exp \left(\frac{-\Delta E_{a}}{R_{g} T}\right) & =\exp \left(\frac{-T_{r}}{\Delta T}\right) \\
& =\exp \left(-\epsilon_{0}\right) \exp \left(\frac{-\phi \theta}{1+\phi}\right),
\end{aligned} \\
& \hat{\Theta}(\eta)=\frac{\phi \theta(\eta)}{1+\phi} .
\end{aligned}
$$


The formulation for the inner zone is completed by the following:

$$
\begin{aligned}
& \frac{d^{2} \hat{\Theta}(\eta)}{d \eta^{2}}= \frac{\operatorname{Le}_{g} \delta^{* 2} \Gamma^{\prime \prime}}{C_{2}{ }^{2}}\left[1-\frac{\Gamma^{\prime \prime \prime}}{C_{2} \Gamma^{\prime}}\right. \\
&\left.-\frac{\Gamma^{\prime \prime \prime}}{C_{2} \Gamma^{\prime}}\left(\frac{1+\phi}{\phi}\right) \frac{d \hat{\Theta}(\eta)}{d \eta}\right] \\
& \times \exp \left(-\epsilon_{0}\right) \hat{\Theta}(\eta) \exp (-\hat{\Theta}), \\
& \hat{\Theta}=\left.\frac{d \hat{\Theta}}{d \eta}\right|_{\eta=0}=0, \quad \eta \rightarrow 0, \\
& \lim _{\eta \rightarrow-\infty} \frac{d \hat{\Theta}(\eta)}{d \eta}=-\frac{\phi}{1+\phi} .
\end{aligned}
$$

The condition for the stoichiometry of the reaction is as follows:

$$
\begin{aligned}
\frac{\Gamma^{\prime \prime \prime}}{C_{2} \Gamma^{\prime}} & =1 \Rightarrow \nu_{o} u_{F}(1-\epsilon)\langle\rho\rangle_{n}{ }^{s} \\
& =\epsilon\left\langle\rho_{o}\right\rangle_{n}^{g}\langle u\rangle_{n}^{g} .
\end{aligned}
$$

The solution to Eq. 51 gives the following expression for the adiabatic temperature $T_{r}$ :

$$
\begin{aligned}
T_{r}^{4} \exp \left(\frac{-\Delta E_{a}}{R_{g} T_{r}}\right) \\
=\frac{\left(\Delta E_{a} \Delta i_{c}\right)^{2}\left(\epsilon\left\langle D_{m}\right\rangle\right)\left(\epsilon\left\langle\rho_{o}\right\rangle_{n}{ }^{g}\langle u\rangle_{n}{ }^{g}\right)^{2}}{\left(R_{g}\langle k\rangle_{l}\right)^{2} a_{1} \nu_{o}{ }^{3}\langle\rho\rangle_{n}{ }^{s}} .
\end{aligned}
$$

The front speed $u_{F}$ is then obtained from Eq. 41 and the integrated form of the energy equation, Eq. 9, which is

$\Delta i_{c}=\frac{\langle\rho\rangle\langle u\rangle c_{p} \nu_{o}\left(T_{r}-T_{n}\right)}{\left\langle\rho_{o}\right\rangle_{n}{ }^{g}\langle u\rangle_{n}{ }^{g} \epsilon}$.

Now, by relaxing the assumption of equal heat capacities for the solid and gas, for stoichiometric combustion, the adiabatic temperature $T_{r, \text { st }}$ becomes

$$
T_{r, \mathrm{st}}=\frac{\Delta i_{c}\left\langle\rho_{o}\right\rangle_{n}^{g}}{\left\langle c_{p}\right\rangle^{g}\left(\left\langle\rho_{o}\right\rangle_{n}{ }^{g}+\nu_{o} \frac{\langle\rho\rangle_{n}{ }^{s}\left\langle c_{p}\right\rangle^{s}}{\left\langle c_{p}\right\rangle^{g}}\right)}+T_{n} .
$$

The stoichiometric inlet, air pore-velocity required for the total consumption of the solid fuel and oxygen can then be determined from the following expression:

$$
\begin{aligned}
\langle u\rangle_{n, \mathrm{st}}{ }^{g}= & \frac{T_{r, \mathrm{st}}{ }^{2} \exp \left(-\Delta E_{a} / 2 R_{g} T_{r, a}\right)\langle k\rangle_{t}}{\left(\Delta E_{a} / R_{g}\right) \sqrt{\epsilon^{3}} \Delta i_{c}\left\langle\rho_{o}\right\rangle_{n}{ }^{g}} \\
& \times \sqrt{\frac{a_{1} \nu_{o}^{3}\langle\rho\rangle_{n}{ }^{3}}{\left\langle D_{m}\right\rangle}} .
\end{aligned}
$$

The front speed for the stoichiometric condition will then be determined from Eq. 54, using $\langle u\rangle_{n, s t}^{g}$ as the air pore-velocity.

\section{Numerical Solution}

The governing energy and species equations, subject to the boundary conditions, Eqs. 33-36, were rewritten for a finite volume, which lead to a system of algebraic equations. Constant properties were assumed, except for the temperature dependence of the radiative conductivity, the gas density, and binary mass diffusion. A nonuniform finite volume distribution was used, with a higher concentration near the combustion front. The power-law scheme [19] described the fluxes at the finite control-volume faces. The species and temperature distributions, a well as the front velocity, were solved iteratively, prescribing an inlet gas flow and a front speed which depended on the final temperature through the integrated energy equation. The reaction rates were linearized to achieve stability during iterations. Variations in gas density and in binary mass diffusion, with respect to temperature and the evolution of the pyrolytic gas, were accounted for. The specific heat and thermal conductivity for air and wood were assumed to be constant.

Since the propagation speed was not known a priori, the location of the front was fixed by specifying the temperature at a point in space (i.e., node). This avoided the cold-boundary difficulty. This node was selected in such a way to ensure that temperature and species gradients nearly vanished at the cold boundary; otherwise, heat loss would occur through this boundary. 
Species distributions were initially computed using an assumed linear temperature distribution. When a converged solution was obtained for the gaseous and the solid species distributions, the energy equation was included in the iteration. The energy equation was solved using the latest species distributions. The convergence criterion, adopted for both the species and temperature distributions, was less than $10^{-9}$. Because of the strong temperature dependence of the reaction rate, as the temperature increased, the source terms became large, causing numerical instabilities. It was therefore necessary to compute the reaction rate only during the iteration on the species equations, keeping it constant while solving the energy equation.

\section{Impact of Uncertainty in Parameters}

Typical numerical solutions for temperature, oxygen, and solid-fuel density distributions, and volumetric oxygen consumption rate, are shown in Fig. 2. The results for the temperature distribution are also compared with our experimental results, which will be described in the following section. In the experiment, the bed

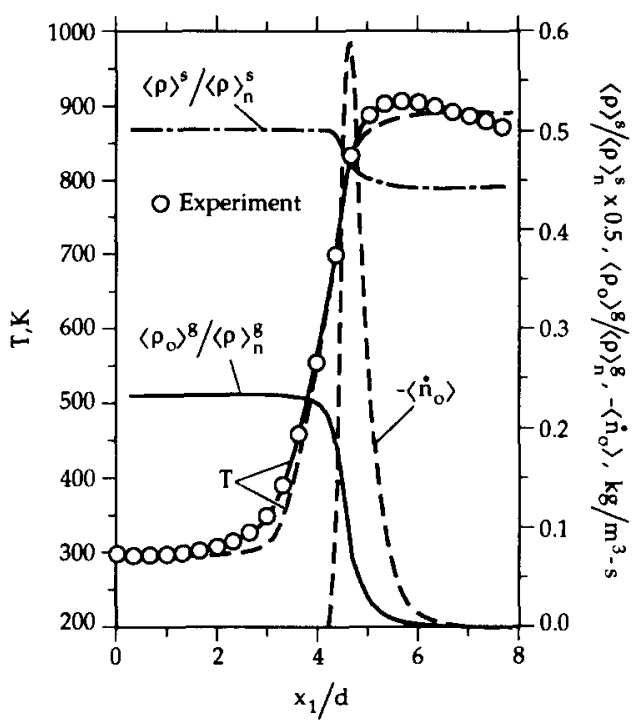

Fig. 2. A typical distribution of the temperature, the dimensionless oxygen and solid fuel densities, and the oxygen consumption rates. The corresponding experimental results for the temperature are also shown. The experimental front speed is used to transform the measured transient temperature variation to the spatial distribution. height was finite, causing possible heat losses to occur from the top of the bed. However, only a thin layer of char-ash is needed to nearly isolate the front from the end heat losses. Given this experimental artifact, the agreement between the experiment and predictions for $T=T(x, t)$ is rather good. The experimental front speed was used to transform the transient local temperature into the quasi-steady spatial distribution. Among prescribed parameters, the most uncertainty can be attributed to the kinetic constants, the preexponential factor $a_{1}$ and the activation energy $\Delta E_{a}$. The effective transport properties are those available in the literature and the predicted results show that they are most sensitive to the reaction kinetic constants. In the literature, several experimental results are available on the kinetic constants of wood and cellulosic materials. Kashiwagi and Nambu [20] determined the first-order global kinetic constants for the three-step reaction mechanism for cellulosic paper (Eqs. 1-3) using a thermogravimetric technique. The parameters they obtained are listed in Table 1. Rogers and Ohlemiller [6, 8] reported $163 \mathrm{~kJ} / \mathrm{mol}$ for the activation energy and $6.7 \times 10^{8} \mathrm{~s}^{-1}$ for the pre-exponential factor. Despite the close agreement between the activation energies, the discrepancy between the pre-exponential factors can be attributed to the difference between the inferred order of the reaction.

The kinetic data reported by Roberts [21] indicate two activation energies of 235 and 126 $\mathrm{kJ} / \mathrm{mol}$ for the pyrolysis of wood. This suggests that pyrolysis may take place by simple or complex kinetics. Shafizadeh [22] presents two alternative pathways for the decomposition of cellulosic materials. These are

Cellulose $\underset{\rightarrow \text { Char }+ \text { Gases }}{\rightarrow}$

TABLE 1

Global Kinetic Constants ${ }^{a}$

\begin{tabular}{lccc}
\hline & Pyrolysis & $\begin{array}{c}\text { Oxidative } \\
\text { Degradation }\end{array}$ & $\begin{array}{c}\text { Char } \\
\text { Oxidation }\end{array}$ \\
\hline$\Delta E_{a}, \mathrm{~kJ} / \mathrm{mol}$ & 220 & 160 & 160 \\
$a_{1}, \mathrm{~s}^{-1}$ & $2 \times 10^{17}$ & $2.5 \times 10^{12}$ & $5.67 \times 10^{9}$ \\
\hline
\end{tabular}

\footnotetext{
${ }^{a}$ Data from Kashiwagi and Nambu [20].
} 
The kinetic rates for volatiles $K_{v}$ and for char $K_{c}$ for this cellulose pyrolysis are reported by Shafizadeh [22], and are as follows:

$K_{v}=3.2 \times 10^{14} \exp \left(-198 / R_{g} T\right)$,

$K_{c}=1.3 \times 10^{10} \exp \left(-151 / R_{g} T\right)$.

Shafizadeh also reports the yields, elemental composition, and empirical formulas of chars obtained by isothermal heating of cellulose, wood, and lignin samples for $5 \mathrm{~min}$ within the temperature range of $300^{\circ}-500^{\circ} \mathrm{C}$. The results are shown in Table 2. It appears that the chemical composition of the remaining char $\mathrm{C}_{6} \mathrm{H}_{4.5} \mathrm{O}_{1.4}$ does not vary with the starting materials. Since the primary elements that compose wood are cellulose and lignin [23], the model of Shafizadeh [22] can be extended to include a third reaction (char oxidation with the kinetics data of Kashiwagi and Nambu [20]) to give

Cellulose $\underset{\rightarrow}{\rightarrow}$ Vhalatiles $_{1}+$ Gases $_{1} \rightarrow \mathrm{Char}_{2}+$ Gases $_{2}$.

We note that the presence of alkali and metal species do influence the kinetics, and therefore, no generalization can be made. Figures $3 a$ and $b$ illustrate the effect of the variations in the pre-exponential factor and the activation energy on the front speed, using the closed-form solutions for Eqs. 55 and 56. The experimental results and their uncertainties are also shown. The thermophysical properties used in the computations are given in Table 3. The heat capacity and thermal conductivity for air (at $300 \mathrm{~K}$ ) and wood are given by Incropera and DeWitt [24], and were assumed to be independent of temperature. The binary mass diffusion coefficient (oxygen-nitrogen) is given by Reid et al. [25]. The amount of oxygen required for the stoichiometric burning of wood $\nu_{o}$ is $1.25 \mathrm{~kg} \mathrm{O}_{2} / \mathrm{kg}$ fuel [26]. The heating value for wood $\Delta i_{c}$, was taken to be $14 \mathrm{MJ} / \mathrm{kg}$ fuel, as reported by Summitt and Sliker [27], and as measured in our experiments (discussed in the following section). It is evident from Fig. $3 \mathrm{~b}$ that a variation in the pre-exponential frequency factor significantly influenced the front speed, whenever the incoming air flow rate was low and the reaction kinetically controlled. For mass diffusion controlled reactions, on the other hand, the activation energy for the mass diffusion dominates, and $a_{1}$ did not influence the front speed.

The effect of the heat of combustion on the front speed is shown in Fig. 3c. The magnitude of $\Delta i_{c}$ for wood varies depending on the type of wood and its moisture content. High moisture content lowers $\Delta i_{c}$ (Summitt and Sliker [27]).

\section{EXPERIMENT}

\section{Apparatus and Procedure}

Figure 4 is a schematic of the packed bed used in the combustion of wood particles. The moisture content corresponded to relative humidity in air of about 50 percent. The experiment is similar to that of Torero et al. [11] for the combustion of polyurethane. The spherical

TABLE 2

Starting Material and Elemental Composition of Its $\mathrm{Char}^{a}$

\begin{tabular}{|c|c|c|c|c|c|c|}
\hline \multirow[b]{2}{*}{ Material } & \multirow{2}{*}{$\begin{array}{c}T \\
\left({ }^{\circ} \mathrm{C}\right)\end{array}$} & \multirow{2}{*}{$\begin{array}{c}\text { Char Yield } \\
\text { (wt.\%) }\end{array}$} & \multicolumn{3}{|c|}{ Composition } & \multirow[b]{2}{*}{ Empirical Formula } \\
\hline & & & $\mathrm{C}$ & $\mathrm{H}$ & $\mathrm{O}$ & \\
\hline \multirow[t]{6}{*}{ Cellulose } & No treatment & - & 42.8 & 6.5 & 50.7 & $\mathrm{C}_{6} \mathrm{H}_{11} \mathrm{O}_{5.3}$ \\
\hline & 325 & 63.3 & 47.9 & 6.0 & 46.1 & $\mathrm{C}_{5} \mathrm{H}_{9} \mathrm{O}_{4.3}$ \\
\hline & 350 & 33.1 & 61.3 & 4.8 & 33.9 & $\mathrm{C}_{6} \mathrm{H}_{5.6} \mathrm{O}_{2.5}$ \\
\hline & 400 & 16.7 & 73.5 & 4.6 & 21.9 & $\mathrm{C}_{6} \mathrm{H}_{4.5} \mathrm{O}_{1.3}$ \\
\hline & 450 & 10.5 & 78.8 & 4.3 & 16.9 & $\mathrm{C}_{6} \mathrm{H}_{3,9} \mathrm{O}_{1.0}$ \\
\hline & 500 & 8.7 & 80.4 & 3.6 & 16.1 & $\mathrm{C}_{6} \mathrm{H}_{3.2} \mathrm{O}_{0.9}$ \\
\hline \multirow[t]{2}{*}{ Wood } & No treatment & 一 & 46.4 & 6.4 & 47.2 & $\mathrm{C}_{6} \mathrm{H}_{9.9} \mathrm{O}_{4.6}$ \\
\hline & 400 & 24.9 & 73.2 & 4.6 & 22.2 & $\mathrm{C}_{6} \mathrm{H}_{4.5} \mathrm{O}_{1.4}$ \\
\hline \multirow[t]{2}{*}{ Lignin } & No treatment & - & 64.4 & 5.6 & 24.8 & $\mathrm{C}_{6} \mathrm{H}_{6.5} \mathrm{O}_{2.0}$ \\
\hline & 400 & 73.3 & 72.7 & 5.0 & 22.3 & $\mathrm{C}_{6} \mathrm{H}_{5.0} \mathrm{O}_{1.3}$ \\
\hline
\end{tabular}

${ }^{a}$ Data from Shafizadeh [22]. 

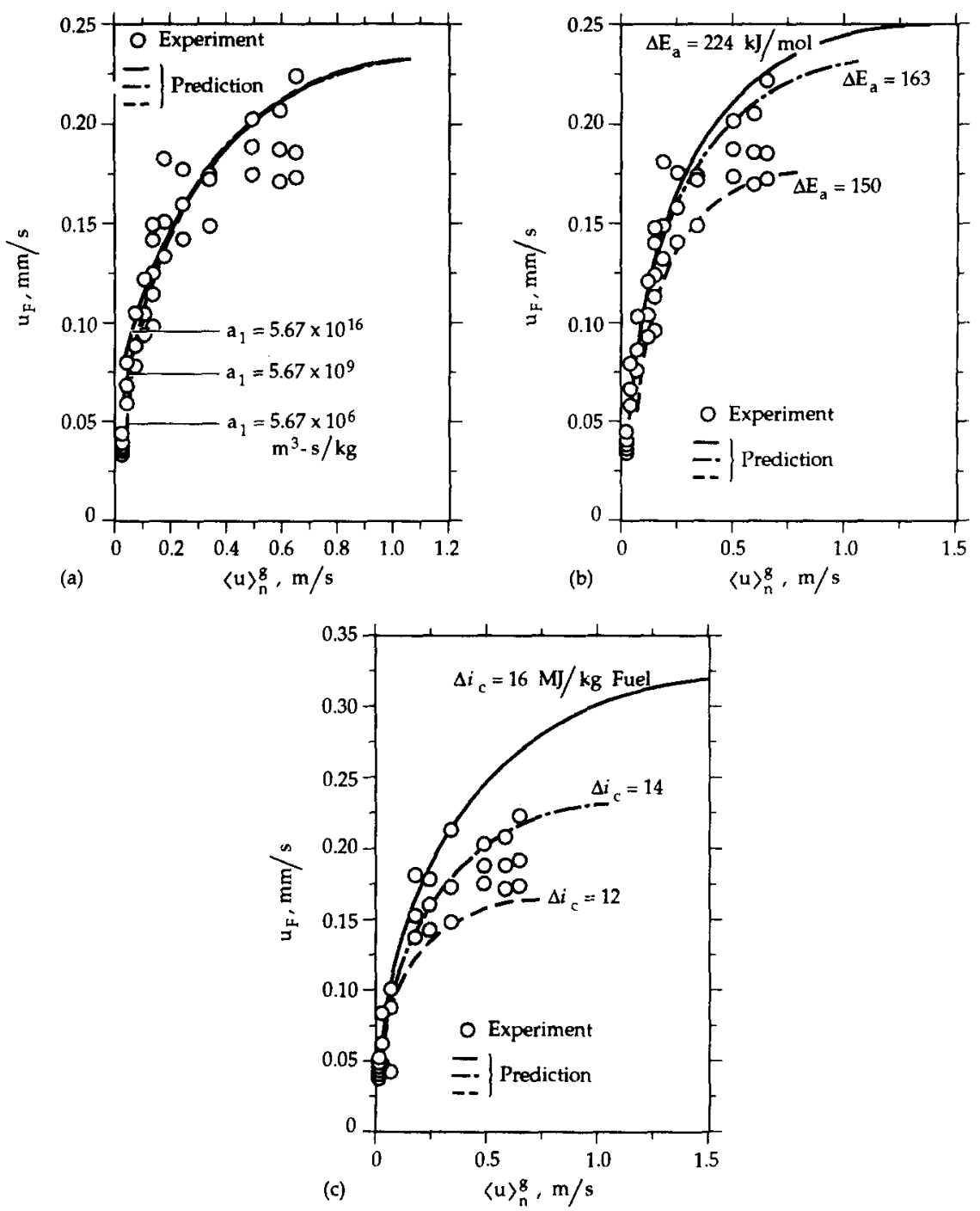

Fig. 3. Effect of the kinetic and thermodynamic parameter uncertainties on the predicted variation of the front speed with respect to the air pore-velocity. (a) Frequency factor, (b) activation energy, and (c) heat of combustion. The experimental results are also shown.

TABLE 3

Thermochemicophysical Properties used in Computations

\begin{tabular}{|c|c|c|c|c|}
\hline & & Gas & Other & Solid \\
\hline$c_{p}$ & $\mathrm{~kJ} / \mathrm{kg}-\mathrm{K}$ & 1.05 & - & 2.81 \\
\hline$k$ & $\mathrm{~W} / \mathrm{m}-\mathrm{K}$ & $26.3 \times 10^{-3}$ & - & 0.15 \\
\hline$\rho$ & $\mathrm{kg} / \mathrm{m}^{3}$ & 1.16 & 一 & 663 \\
\hline$D_{m, g}$ & $\mathrm{~m}^{2} / \mathrm{s}$ & $2.1 \times 10^{-5}$ & - & 一 \\
\hline$\Delta i_{c}$ & $\mathrm{MJ} / \mathrm{kg}$ fuel & - & 14 & 一 \\
\hline$\nu_{o}$ & $\mathrm{~kg} \mathrm{O}_{2} / \mathrm{kg}$ fuel & - & 1.25 & - \\
\hline$F$ & - & - & 0.68 & - \\
\hline$d$ & $\mathrm{~m}$ & - & $6.4 \times 10^{-3}$ & 一 \\
\hline$\Delta E_{a}$ & $\mathrm{~kJ} / \mathrm{mol}$ & 一 & 163 & 一 \\
\hline$a_{1}$ & $\mathbf{s}^{-1}$ & - & $5.67 \times 10^{9}$ & 一 \\
\hline
\end{tabular}

wood particles (diameter $6.4 \times 10^{-3} \mathrm{~m}$ ) were packed randomly. The chamber had a square cross-sectional area $\left(A=5.81 \times 10^{-3} \mathrm{~m}^{2}\right)$, and was made of very low porosity $80 \%$ alumina-20\% silica to minimize lateral heat losses. The length of the combustion chamber was $0.14 \mathrm{~m}$. Air was supplied from below (opposite to the front propagation). The Darcean velocity $\epsilon\langle u\rangle_{n}{ }^{g}$ was determined using the measured volumetric flow rate and the cross-sectional area. The flow meters are precalibrated. Because of the presence of upstream mixtures and valves, no noticeable change in the air flow 


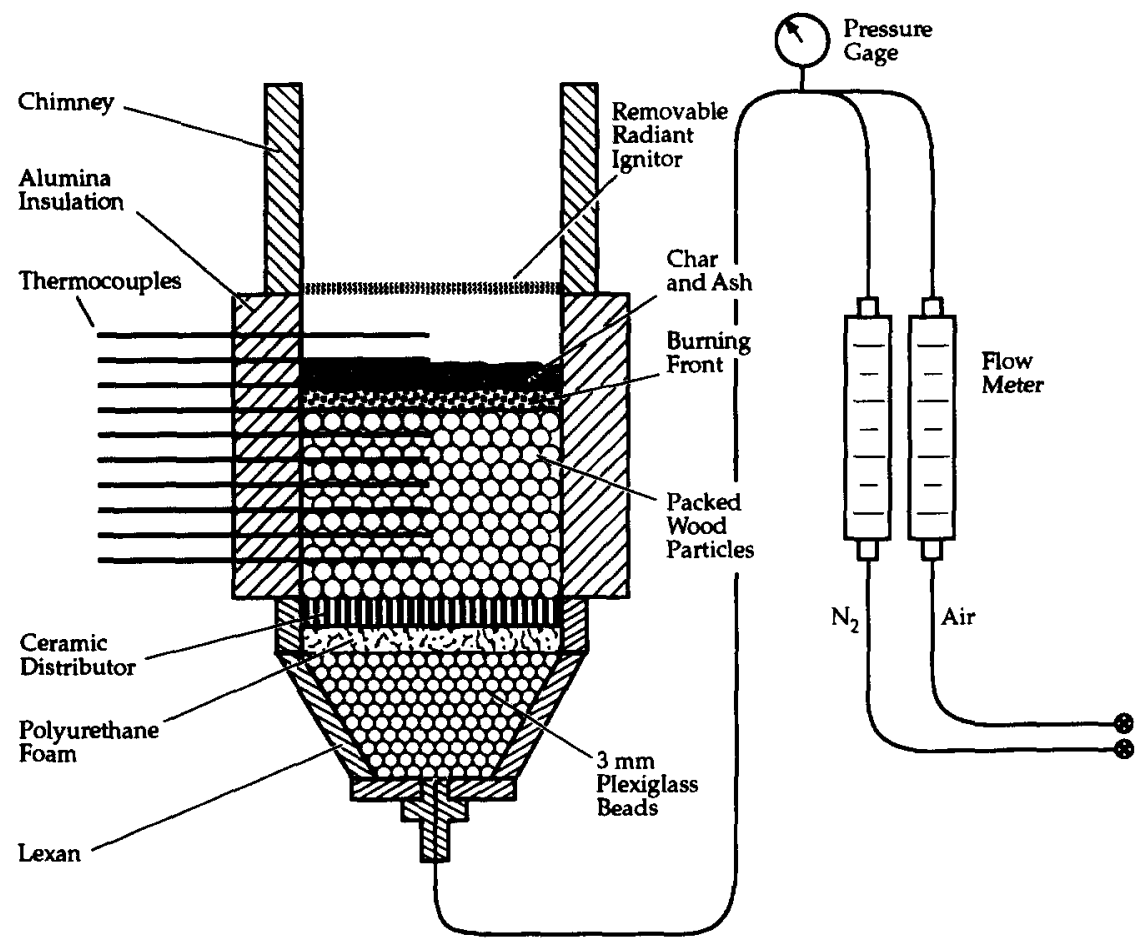

Fig. 4. A schematic of the experiment showing the packed bed, the air flow, and the thermocouples.

rate was observed when the combustion of the packed bed began. For mixers, Plexiglas beads and a polyurethane foam are used upstream to insure flow uniformity, and to minimize channeling. The wood particles on the top layer were uniformly ignited from above using a radiant heat source. The combustion front propagated downward at a nearly steady rate and opposite to the direction of the air flow.

A chimney, $0.3 \mathrm{~m}$ in length, was placed on top of the combustion chamber to eliminate air reaching the front from above. For small $\langle u\rangle_{n}{ }^{g}$,-a thick layer of unburned char residue formed above the reaction zone (i.e., incomplete reaction) as the front spread downward. This further diminished air diffusion from the downstream and thermally insulated the front. For large $\langle u\rangle_{n}^{g}$, substantial flaming occurred throughout the chimney. The heat of combustion was measured using a bomb calorimeter (Parr 1108 Oxygen Combustion Bomb) and was $14 \mathrm{MJ} / \mathrm{kg}$ with $\pm 4 \%$. Note that in the bomb calorimeter the entire combustible matter is consumed. In the reverse combustion experiment not all the wood volatiles (which vary with the extent of gasification) yield $\mathrm{CO}_{2}$ and $\mathrm{H}_{2} \mathrm{O}$ and the same amount of heat. This becomes more significant when the adiabatic (or maximum) temperature is rather low, and a substantial amount of $\mathrm{CO}$ and tars are produced.

Temperature was measured using B-type ( $\mathrm{Pt}$ $30 \% \mathrm{Rh} / \mathrm{Pt} 6 \% \mathrm{Rh}$ ) thermocouples (insulated in ceramic tubes) inserted laterally along the centerline of the bed. The thermocouples were spaced $9.5 \times 10^{-3} \mathrm{~m}$ apart, recorded every 10 s. The front speed was determined from this known spacing, using the time for a given temperature, e.g., $500^{\circ} \mathrm{C}$, to be recorded by two successive thermocouples. The results show that the front speed was essentially constant.

In a few cases, thermocouples were also placed within the particles and some differences noted with the unshielded thermocouple in the gas phase. In principle, the onedimensional temperature distribution within the front is expected to be slightly different for the gas and solid phases, especially at high $\langle u\rangle_{n}{ }^{g}$. This is because of the different gas and solid-surface reactions and kinetics. The mea- 
sured temperature by the thermocouples in the gas phase was considered to be an average pore-solid temperature, because of radiative heat transfer with the particles and conductive heat transfer with the char particles.

\section{Results}

Figure 5 shows some typical temperature recordings throughout the bed; nearly steadystate propagation is apparent. The lower maximum temperature for the thermocouple at the uppermost location can be attributed to the radiation heat loss. At low flow rates, as the front moved inward, the accumulation of char and ash layers insulated the front. However, as the reaction front moved downward, the bed height decreased because of particle shrinkage. The observed rapid drop in the temperature indicated that the thermocouple was no longer located in the bed, i.e., it was exposed to the gaseous products of the combustion.

As evident in Fig. 5, the maximum temperature changes slightly as the front moves into the bed and approaches the bottom of the bed. Here no attempt has been made to distinguish among the top, middle, and bottom sections of the bed, and an average is taken for the middle section. Torero et al. [11] make such a distinction and points out to different regimes prevailing in these sections.

To determine the regimes of flow and reaction in the packed bed, the volumetric air flow rate was varied between $4 \times 10^{-5}$ and $1.6 \times$

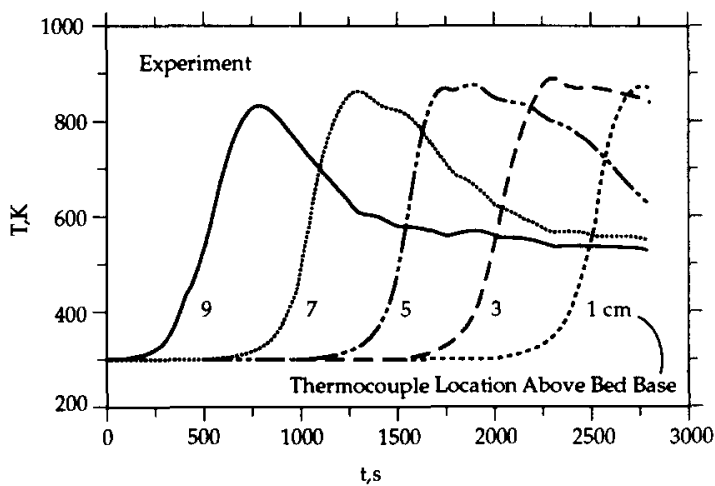

Fig. 5. The measured variation of the local temperature with respect to time, for thermocouples located at various locations above the base of the bed.
$10^{-3} \mathrm{~m}^{3} / \mathrm{s}$. At very low flow rates, the bed would not ignite, defining a lower flammability limit. Also, for air flow rates above $8 \times 10^{-4}$ $\mathrm{m}^{3} / \mathrm{s}$, fluidization of the bed occurred. The experimentally determined regime diagram is shown in Fig. 6, and represents the variation of the front speed with respect to the air polevelocity. The scatter in the experimental data shows variation in $u_{F}$ found among the top, middle, and bottom portions of the bed. Heat losses in the top and bottom portions were expected to be significant. The upper and lower limits for ignition are also shown in Fig. 6. The scatter in the experimental data is also partly because of the slight displacement of the thermocouple beads from their initial positions. Torero et al. [11] also observe a decrease in $u_{F}$ at high $\langle u\rangle_{n}{ }^{g}$ and also show that upon preheating the incoming air, the upper $\langle u\rangle_{n}{ }^{g}$ extinction limit can be increased.

\section{DISCUSSION}

\section{Oxygen- versus Fuel-Limited Regime}

For adiabatic combustion, the front speed was essentially controlled by the heat generated in the reaction zone and the transport of this heat to the unburned solid. Thus, radiation is an important mechanism in controlling front speed. The final temperature and the extent of solid consumption depend on the front speed, which is not known a priori. At low air flow rates, the reaction was oxygen limited and the

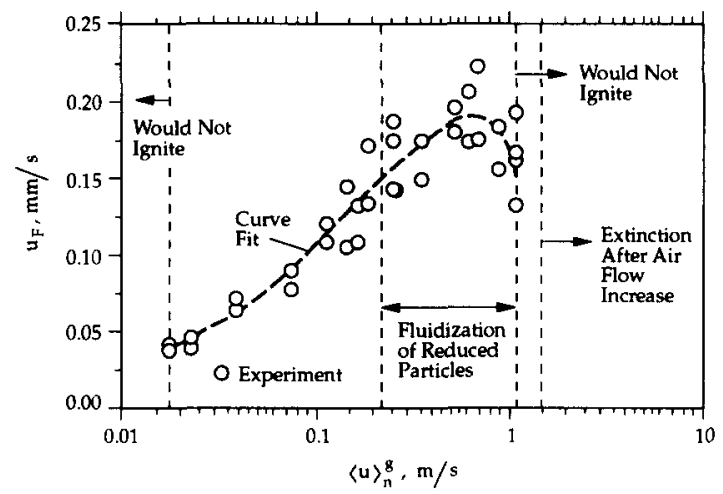

Fig. 6. Experimentally obtained regime diagram showing the propagation of the burning front with respect to the air pore-velocity. The limits of extinction and fluidization are also shown. 
extent of solid conversion to $\langle\rho\rangle_{r}^{s} /\langle\rho\rangle_{n}{ }^{s}$ was small. Increasing the air flow rate resulted in a higher burning velocity, accompanied by a higher temperature, and a larger extent of solid conversion. Figure 7 shows the increase in the extent of solid conversion as the inlet air pore-velocity $\langle u\rangle_{n}{ }^{g}$ increases, as was predicted by the analysis. Beyond a critical flow (corresponding to the stoichiometric burning, where both solid and oxygen are totally consumed), the solid was totally consumed, and an excess of oxygen remained (i.e., fuel-limited regime). Within the fuel-limited regime, increasing the air flow rate resulted in a temperature lower than that corresponding to the stoichiometric burning, and thus a lower front speed. This is primarily because of the convective cooling of the reaction front by the excess oxygen. Within the oxygen-limited regime, increasing $\langle u\rangle_{n}{ }^{g}$ resulted in a higher adiabatic temperature and a higher burning speed.

In Fig. 7, the axial location is scaled with respect to particle diameter. As shown, the dimensionless front thickness increased with increases in $\langle u\rangle_{n}{ }^{g}$. Figures $8 \mathrm{a}$ and $8 \mathrm{~b}$ show the numerical solutions for the variation of the adiabatic temperature and front speed with respect to $\langle u\rangle_{n}{ }^{g}$, in both the oxygen- and fuellimited regimes. The boundary between the

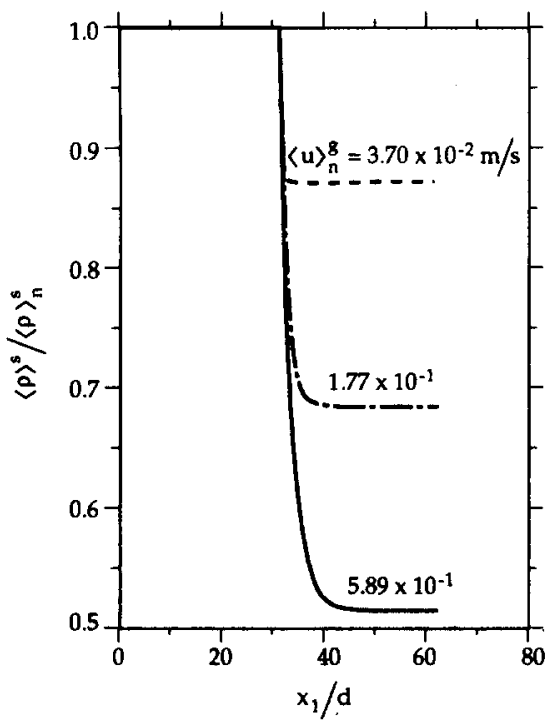

Fig. 7. The predicted spatial variation of the normalized solid density (i.e., conversion ratio) for various air porevelocities. two regimes is marked by the stoichiometric burning [Fig. 8b]. As was shown in Fig. 6, this was above the point of incipient fluidization for the wood particles used $\left(d=6.4 \times 10^{-3} \mathrm{~m}\right)$. Here, the experimental results are comparable to the predicted results. The experimental results shown in Figs. 2, 3, 5, and 9 correspond to no significant fluidization.

The behavior in the oxygen-limited regime observed here is similar to that observed and predicted for reverse smoldering by Dosanjh et al. [10], Ohlemiller et al. [28], and Torero et al. [11], for forward smoldering of polyurethane foams. For very low air flow rates, Dosanjh et al. [10] predict a linear rise in the adiabatic temperature with respect to the air flow rate. This is similar to the trend found in Fig. 8a.

\section{Front Speed and Extinction Limits}

Figures $9 \mathrm{a}$ and $9 \mathrm{~b}$ show the variations of the maximum temperature and front speed with respect to the incoming air pore-velocity for the oxygen-limited regime, where a comparison can be made with experimental results. Thermophysical properties are listed in Table 3. As shown in Fig. 6, the upper extinction limit is about $\langle u\rangle_{n}{ }^{g}=1.4 \mathrm{~m} / \mathrm{s}$. Despite the uncertainty in the experimental results, good agreement is found between the predicted and experimental results for $T_{r}$ and $u_{F}$. At the stoichiometric point, with $\langle u\rangle_{n}{ }^{g}=1.1 \mathrm{~m} / \mathrm{s}, T_{r}$ is about $1920 \mathrm{~K}$ and $u_{F} 0.23 \mathrm{~m} / \mathrm{s}$. Near the stoichiometric point, the numerical and the asymptotic results are in close agreement. At lower values of $\langle u\rangle_{n}^{g}$, the numerical results predict higher $T_{r}$ and $u_{F}$, which are in closer agreement with the experimental results.

A distinction should be made between the point where the front ceases to propagate in a fully ignited bed (extinction) and the point where the bed would not ignite. In the latter case, the top layer of the packed bed was ignited via the radiation from an external heat source, which, upon removal, extinguished the bed. This can be attributed to the heat losses at the boundary. Based on the numerical computations, the extinction limit was determined to be $\langle u\rangle^{g}=1.8 \mathrm{~m} / \mathrm{s}$ (Fig. 8b). The upper extinction limit was determined experimentally by allowing for the reaction front to propagate 


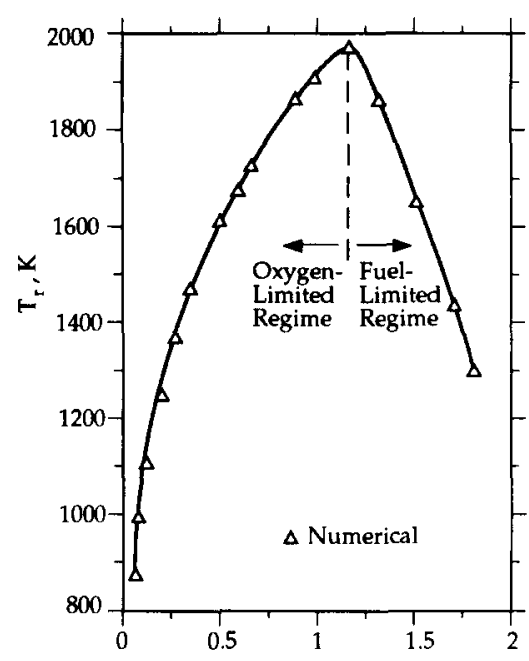

(a)

$\langle u\rangle_{n}^{8}, \mathrm{~m} / \mathrm{s}$

Fig. 8. The predicted effect of the air pore-velocity on (a) the adiabatic temperature, and (b) the front speed, for the oxygen- and fuel-limited regimes.

deep within the bed (at a low air flow rate) and then gradually increasing the air flow rate to the point where the burning front ceased to propagate. This point was above the incipient fluidization of the bed, thus resulting in the loss of the burning particles from the combustion zone. There, no solid or ash residue accumulated downstream of the front to minimize radiation losses. Since the analysis assumed adiabatic conditions, the upper extinction point

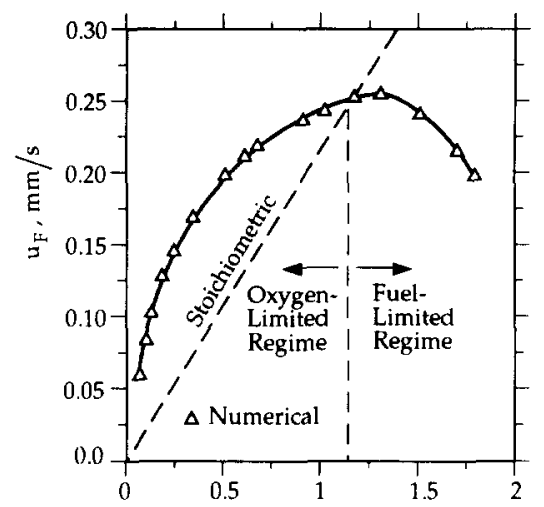

(b)

$\langle u\rangle_{n}^{g}, m / s$ predicted numerically is higher than that obtained experimentally.

\section{Validity of Volume-Averaged Treatment}

The local volume-averaged treatment assumed that the variation of temperature and concentration across the representative elementary volume was much smaller than that across the system. For an ordered arrangement of spheri-
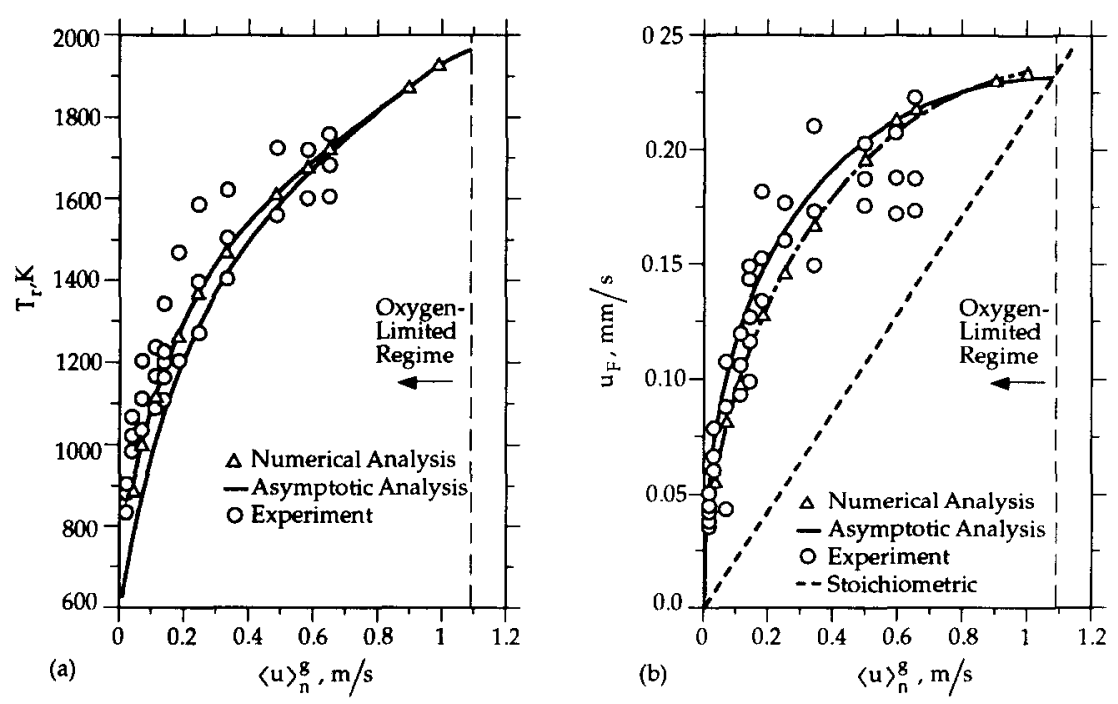

Fig. 9. Variation of (a) the adiabatic temperature, and (b) the front speed with the air pore-velocity for the oxygen-limited regime. The experimental and predicted (numerical and asymptotic) results are shown as well as the results based on stoichiometric burning. 
cal particles, a minimum of one particle must be contained in the representative elementary volume. For the volume-averaged treatment to remain valid here, the front thickness should be larger than a few particles. The width of the front was determined numerically and compared with the initial particle diameter $d$. The front thickness was defined as the length of the medium across which the reaction rate was equal or greater than $1 / 10$ of its maximum value. Figure 10 shows the variation of the normalized front thickness with respect to the pore velocity. The higher air flow rate results in a wider front, which can be attributed to the larger total diffusion coefficients for heat and mass transport. As the air flow rate increases, the axial hydrodynamic dispersion coefficients become larger than the molecular diffusion coefficients, resulting in higher total mass and thermal diffusivities. Furthermore, the energy transport via radiation also becomes more significant (since higher temperatures are achieved as the incoming air flow rate is increased). As is evident from Fig. 10, the volume-averaged treatment is expected to become questionable for low air pore-velocities, when the front thickness becomes nearly the same as the particle diameter. In this case, a two-medium treatment, or a direct pointwise simulation, may be necessary. This has been

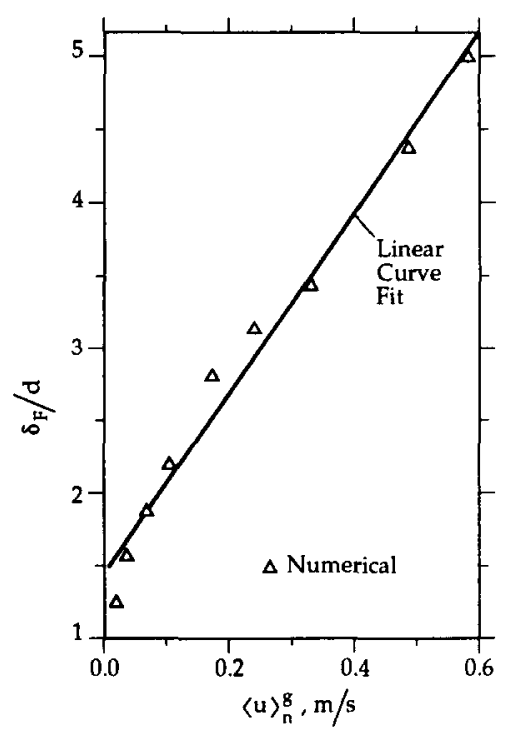

Fig. 10. Variation of the predicted normalized front thickness with respect to the air pore-velocity. done for the premixed, gas-phase reactions in otherwise inert porous media [29].

\section{SUMMARY}

Burning of a packed bed of wood particles in a reverse combustion with air flowing from below was modeled using single-step reaction kinetics, which assumed that the surface char oxidation was dominant. Local thermal equilibrium and local chemical nonequilibrium between the solid and gas phases were assumed. Both the oxygen- and fuel-limited regimes were considered. Supporting experiments were also performed. At low air flow rates, where the particles were not fluidized or a layer of ash and partly consumed wood particles remained on top of the bed, predictions for the oxygenlimited regime agreed with experimental results.

In the oxygen-limited regime, an increase in the air flow rate increased the adiabatic temperature. This was accompanied by an increase in consumption, which produced more pyrolytic gases and ash. Higher temperatures in the reaction zone triggered gas-phase reactions. In this regime, the front speed also increased with an increase in the air flow rate. However, the speed was higher than the corresponding stoichiometric speed. Oxygen was totally consumed in the reaction zone and char remained nonconsumed after the passage of the front.

In the fuel-limited regime, both the adiabatic temperature and front speed decreased as the air flow rate was increased. This regime can be characterized by total solid consumption and an excess oxygen flow (i.e., oxygen leak) downstream of the front. The front velocity was lower than the stoichiometric burning velocity.

The air flow required for stoichiometric burning of the solid depends on the kinetic constants of the reaction and is directly proportional to the first power of the total conductivity of the medium.

In the fuel-limited regime, by monotonically increasing the air velocity, an upper extinction limit was reached beyond which the front would not propagate through the medium (due to the excessive cooling of the reaction front). In the 
experiments, a lower extinction limit was identified for the self-sustained propagation of the reaction front. Below this limit, the fraction of solid burned was too small to produce the heat required for preheating the incoming gas and initiating the reactions.

We would like to thank Mr. S. Turner for performing the experiments, and Dr. K. Hanamura for valuable discussions.

\section{REFERENCES}

1. Palmer, K. M., Combust. Flame 1:129-154 (1957).

2. Ohlemiller, T. J., Smoldering Combustion Hazards of Thermal Insulation Materials, National Bureau of Standards, Washington, D.C., 1981.

3. Ohlemiller, T. J., Combust, Flame 81:341-353 (1990).

4. Ohlemiller, T. J., Prog. Ener. Combust. Sci. 11:277-310 (1985).

5. Ohlemiller, T. J., Combust. Flame 81:354-365 (1990).

6. Rogers, F. E., and Ohlemiller, T. J., J. Fire Flammability 11:32-44 (1980)

7. Ohlemiller, T. J., and Rogers, F. E., J. Fire Flammability 9:489-509 (1978).

8. Rogers, F. E., and Ohlemiller, T. J., Combust. Sci. Technol. 24:129-137 (1980).

9. Robinovich, O. S., and Gurevich, F. G., Int. J. Heat Mass Transf. 29:241-255 (1986).

10. Dosanjh, S. S., Pagni, P. J., and Fernandez-Pello, A. C., Combust. Flame 68:131-142 (1987).

11. Torero, J. L., Fernandez-Pello, A. C., and Kitano, M., Combust. Sci. Technol. 91:95-117 (1993).

12. Ohlemiller, T. J., and Lucca, D. A., Combust. Flame 54:131-147 (1983).
13. Moussa, N. A., Toong, T. Y., and Garris, C. A., Sixteenth Symposium (International) on Combustion, The Combustion Institute, Pittsburgh, 1977, p. 1447.

14. Glassman, I., Combustion, Academic, New York, 1987.

15. Wakao, N., and Kaguei, S., Heat and Mass Transfer in Packed Beds, Gordon and Breach, New York, 1982.

16. Kaviany, M., Principles of Heat Transfer in Porous Media, Springer-Verlag, New York, 1991.

17. Singh, B. P., and Kaviany, M., Int. J. Heat Mass Transf. (in press).

18. Britten, J. A., and Krantz, W. B., Combust. Flame 60:125-140 (1985).

19. Patankar, S. V., Numerical Heat Transfer and Fluid Flow, Hemisphere, Washington, D.C., 1980.

20. Kashiwagi, T., and Nambu, H., Combust. Flame 88:345-368 (1992).

21. Roberts, F. E., Combust, Flame 14:261-272 (1970).

22. Shafizadeh, F., in The Chemistry of Solid Wood (R. Rowell, Ed.), ACS, Washington, 1984, p. 491.

23. Parker, W. J., Ph.D. dissertation, George Washington University, 1988.

24. Incropera, F. P., and Dewitt, D. P., Introduction to Heat Transfer, 2nd ed., Wiley, New York, 1985.

25. Reid, R. C., Prausnitz, J. M., and Poling, B. E., The Properties of Gases and Liquids, 4th ed., McGraw-Hill, New York, 1987.

26. Tillman, D. A., Rossi, A. J., and Kitto, W. D., Wood Combustion: Principles, Processes, and Economics, Academic, New York, 1981.

27. Summitt, R., and Sliker, A., Handbook of Materials Science, CRC Press, New York, 1980.

28. Ohlemiller, T. J., Bellan, J., and Rogers, F. E., Combust. Flame 36:197-215 (1979).

29. Sahraoui, M., and Kaviany, M., Int. J. Heat Mass Transf, (in press).

Received 22 November 1993; revised 26 April 1994 